\title{
On the Incidence Energy of Some Toroidal Lattices
}

\author{
Jia-Bao Liu,, ${ }^{1,2}$ Jinde Cao, ${ }^{2,3}$ and Jin Xie ${ }^{4}$ \\ ${ }^{1}$ Department of Public Courses, Anhui Xinhua University, Hefei 230088, China \\ ${ }^{2}$ Department of Mathematics, Southeast University, Nanjing 210096, China \\ ${ }^{3}$ Department of Mathematics, Faculty of Science, King Abdulaziz University, Jeddah 21589, Saudi Arabia \\ ${ }^{4}$ Department of Mathematics \& Physics, Hefei University, Hefei, 230601, China
}

Correspondence should be addressed to Jinde Cao; jdcao@seu.edu.cn

Received 28 May 2014; Accepted 18 July 2014; Published 1 September 2014

Academic Editor: Jun $\mathrm{Hu}$

Copyright ( $\odot 2014$ Jia-Bao Liu et al. This is an open access article distributed under the Creative Commons Attribution License, which permits unrestricted use, distribution, and reproduction in any medium, provided the original work is properly cited.

The incidence energy $\operatorname{IE}(G)$, defined as the sum of the singular values of the incidence matrix of $G$, is a much studied quantity with well known applications in chemical physics. In this paper, we derived the closed-form formulae expressing the incidence energy of the 3.12.12 lattice, triangular kagomé lattice, and $S(m, n)$ lattice, respectively. Simultaneously, the explicit asymptotic values of the incidence energy in these lattices are obtained by utilizing the applications of analysis method with the help of software calculation.

\section{Introduction}

A general problem of interest in physics, chemistry, and mathematics is the calculations of the energy of graphs [13], which has now become a popular topic of research; however, almost all of literature deal with the energy of the finite graphs. Yan and Zhang [4] first considered the asymptotic energy of the infinite lattice graphs; they obtained the asymptotic formulae for energies of various lattices. Historically in lattice statistics, the hexagonal lattice, 3.12.12 lattice, triangular kagomé lattice, and $3^{3} \cdot 4^{2}$ lattice have attracted the most attention [4-9]. Ising spins and XXZ/Ising spins on the $\operatorname{TKL}(m, n)$ have been studied in $[10,11]$.

Let $G$ be a simple graph with $n$ vertices, let $A(G)$ be the adjacency matrix, and let $D(G)$ be the diagonal matrix of vertex degrees of $G$, respectively. The Laplacian eigenvalues of $G$ are $L(G)=D(G)-A(G)$ and the signless Laplacian matrix is $Q(G)=D(G)+A(G)$. The characteristic polynomial $P_{G}(x)=\operatorname{det}\left(x I_{n}-A(G)\right)\left(\right.$ resp., $L_{G}(x)=\operatorname{det}\left(x I_{n}-L(G)\right)$, $Q_{G}(x)=\operatorname{det}\left(x I_{n}-Q(G)\right)$ ) of $A(G)$ (resp., $L(G), Q(G)$ ) is called the $A(G)$ (resp., $L(G), Q(G)$ ) characteristic polynomial or $A(G)$ (resp., $L(G), Q(G)$ ) polynomial of $G$ and is denoted by $A_{G}(x)$ (resp., $L_{G}(x), Q_{G}(x)$ ). The spectrum of $A(G)$ (resp., $L(G), Q(G)$ ) which consists of the $A(G)$ (resp., $L(G), Q(G)$ ) eigenvalues is also called the $A(G)$ (resp., $L(G), Q(G)))$ ) spectrum of $G$, respectively. It is well known that $A(G), L(G)$, and $Q(G)$ are symmetric and positive semidefinite; then we denote the eigenvalues of $A(G), L(G)$, and $Q(G)$ by $\lambda_{1}(G) \geq \lambda_{2}(G) \geq \cdots \geq \lambda_{n}(G)$, $\mu_{1}(G) \geq \mu_{2}(G) \geq \cdots \geq \mu_{n}(G)=0$, and $q_{1}(G) \geq q_{2}(G) \geq \cdots \geq$ $q_{n}(G) \geq 0$, respectively. Details on its theory can be found in recent papers [12-14] and the references cited therein.

The famous graph energy $E(G)$ for a simple graph $G$, introduced by Gutman [1], is defined as $E(G)=\sum_{i=1}^{n}\left|\lambda_{i}\right|$. The quantity can be used to estimate the total $\pi$-electron energy in conjugated hydrocarbons. As an analogue of $E(G)$, the incidence energy $\operatorname{IE}(G)$, is a novel topological index, inspired by Nikiforov idea [2], Jooyandeh et al. [15] introduced the concept $\operatorname{IE}(G)$ of a graph $G$ as $\operatorname{IE}(G)=\sum_{i=1}^{n} \sqrt{q_{i}}$, which is the sum of the singular values of the incidence matrix $B(G)$. The index has attracted extensive attention due to its wide applications in physics, chemistry, graph theory, and so forth; for more work on $\operatorname{IE}(G)$, the readers are referred to papers [15-18].

In $[4,19]$ the energy $E(G)$ and Kirchhoff index $\operatorname{Kf}(G)$ of toroidal lattices were studied. It is an interesting problem to study the incidence energy of some lattices with toroidal boundary condition. Motivated by results above, we consider the problem of computations of the $\operatorname{IE}(G)$ of the 3.12.12 lattice, triangular kagomé lattice, and $S(m, n)$ lattice with toroidal condition in this paper. 


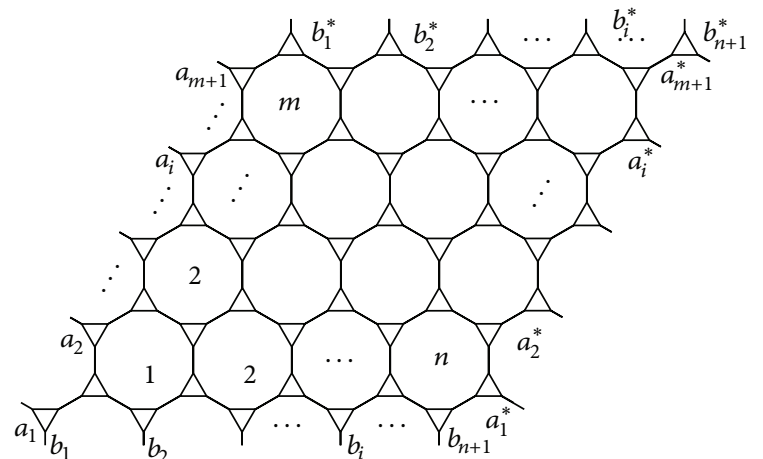

FIgURE 1: The $J^{t}(m, n)$ lattice with toroidal boundary condition [5].

\section{Main Results}

2.1. The 3.12.12 Lattice. The 3.12.12 lattice with toroidal boundary condition by physicists [5], denoted by $J^{t}(m, n)$, is illustrated in Figure 1.

Recently, the adjacency spectrum of 3.12.12 lattice has been proposed in [5] as follows.

Theorem 1 (see [5]). Let $J^{t}(m, n)$ be the 3.12 .12 lattice with toroidal boundary condition. Then the adjacency spectrum is

$$
\begin{aligned}
& \operatorname{Spec}_{A}\left(J^{t}(m, n)\right) \\
& =\{\underbrace{-2,-2, \ldots,-2}_{(m+1)(n+1)}, \underbrace{0,0, \ldots, 0}_{(m+1)(n+1)}\} \\
& \cup\left\{\frac{1 \pm \sqrt{13 \pm 4 \sqrt{3+2 \cos \alpha_{i}+2 \cos \beta_{j}+2 \cos \left(\alpha_{i}+\beta_{j}\right)}}}{2}\right\},
\end{aligned}
$$

where $\alpha_{i}=2 \pi i /(m+1), \beta_{j}=2 \pi j /(n+1), i=0,1, \ldots, m$, $j=0,1, \ldots, n$.

The following result is an important relationship between $\operatorname{Spec}_{A}(G)$ and $\operatorname{Spec}_{Q}(G)$.

Consider that if $G$ is an $r$-regular graph of order $n$, then

$$
D(G)=r I_{n}
$$

Consequently,

$$
Q(G)=D(G)+A(G)=D(G)+r I_{n} .
$$

One can conclude that

$$
Q_{G}(x)=P_{G}(x-r) .
$$

Define the mapping $\varphi\left(\lambda_{i}\right)=\lambda_{i}+r$ maps the eigenvalues of $A(G)$ to the eigenvalues of $Q(G)$ and can be considered as an isomorphism of the $A$-spectrum to the corresponding the Qspectrum for regular graphs.
Suppose that $G$ is an $r$-regular graph with $n$ vertices and $\operatorname{Spec}_{A}(G)=\left\{\lambda_{1}, \lambda_{2}, \ldots, \lambda_{n}\right\}$. Then

$$
\operatorname{Spec}_{Q}(G)=\left\{r+\lambda_{1}, r+\lambda_{2}, \ldots, r+\lambda_{n}\right\}
$$

Note that $J^{t}(m, n)$ is the line graph of the subdivision of $H^{t}(n, m)$ which is a 3 -regular graph with $2(m+1)(n+1)$ vertices, and $J^{t}(m, n)$ has $6(m+1)(n+1)$ vertices. Hence, we get the following theorem.

Theorem 2. Let $J^{t}(m, n)$ be the 3.12.12 lattice with toroidal boundary condition and $\alpha_{i}=2 \pi i /(m+1), \beta_{j}=2 \pi j /(n+1)$, $i=0,1, \ldots, m, j=0,1, \ldots, n$. Then the signless Laplacian spectrum is

$$
\begin{aligned}
& \operatorname{Spec}_{Q}\left(J^{t}(m, n)\right) \\
& =\{\underbrace{1,1, \ldots, 1}_{(m+1)(n+1)}, \underbrace{3,3, \ldots, 3}_{(m+1)(n+1)}\} \\
& \cup\left\{\frac{7 \pm \sqrt{13 \pm 4 \sqrt{3+2 \cos \alpha_{i}+2 \cos \beta_{j}+2 \cos \left(\alpha_{i}+\beta_{j}\right)}}}{2}\right\} .
\end{aligned}
$$

By the definition of the incidence energy, we can easily get the incidence energy of $J^{t}(m, n)$.

Theorem 3. Let $\alpha_{i}=2 \pi i /(m+1), \beta_{j}=2 \pi j /(n+1)$, $i=0,1, \ldots, m, j=0,1, \ldots, n$. Then the incidence energy of $J^{t}(m, n)$ can be expressed as

IE $(J(m, n))$

$$
\begin{aligned}
& =(\sqrt{3}+1)(m+1)(n+1) \\
& +\frac{1}{2} \sum_{i=0}^{m} \sum_{j=0}^{n} \sqrt{7-\sqrt{13-4 \sqrt{3+2 \cos \alpha_{i}+2 \cos \beta_{j}+2 \cos \left(\alpha_{i}+\beta_{j}\right)}}}
\end{aligned}
$$$$
+\frac{1}{2} \sum_{i=0}^{m} \sum_{j=0}^{n} \sqrt{7-\sqrt{13+4 \sqrt{3+2 \cos \alpha_{i}+2 \cos \beta_{j}+2 \cos \left(\alpha_{i}+\beta_{j}\right)}}}
$$$$
+\frac{1}{2} \sum_{i=0}^{m} \sum_{j=0}^{n} \sqrt{7+\sqrt{13-4 \sqrt{3+2 \cos \alpha_{i}+2 \cos \beta_{j}+2 \cos \left(\alpha_{i}+\beta_{j}\right)}}}
$$$$
+\frac{1}{2} \sum_{i=0}^{m} \sum_{j=0}^{n} \sqrt{7+\sqrt{13+4 \sqrt{3+2 \cos \alpha_{i}+2 \cos \beta_{j}+2 \cos \left(\alpha_{i}+\beta_{j}\right)}}} .
$$ 
From theorem above, we consider that

$$
\begin{aligned}
& \lim _{m \rightarrow \infty} \lim _{n \rightarrow \infty} \frac{\operatorname{IE}\left(J^{t}(m, n)\right)}{6(m+1)(n+1)} \\
& =\lim _{m \rightarrow \infty} \lim _{n \rightarrow \infty} \frac{1}{12(m+1)(n+1)} \\
& \times \sum_{i=0}^{m} \sum_{j=0}^{n} \sqrt{7-\sqrt{13-4 \sqrt{3+2 \cos \alpha_{i}+2 \cos \beta_{j}+2 \cos \left(\alpha_{i}+\beta_{j}\right)}}} \\
& +\lim _{m \rightarrow \infty} \lim _{n \rightarrow \infty} \frac{1}{12(m+1)(n+1)} \\
& \times \sum_{i=0}^{m} \sum_{j=0}^{n} \sqrt{7-\sqrt{13+4 \sqrt{3+2 \cos \alpha_{i}+2 \cos \beta_{j}+2 \cos \left(\alpha_{i}+\beta_{j}\right)}}} \\
& +\lim _{m \rightarrow \infty} \lim _{n \rightarrow \infty} \frac{1}{12(m+1)(n+1)} \\
& \times \sum_{i=0}^{m} \sum_{j=0}^{n} \sqrt{7+\sqrt{13-4 \sqrt{3+2 \cos \alpha_{i}+2 \cos \beta_{j}+2 \cos \left(\alpha_{i}+\beta_{j}\right)}}} \\
& +\lim _{m \rightarrow \infty} \lim _{n \rightarrow \infty} \frac{1}{12(m+1)(n+1)} \\
& \times \sum_{i=0}^{m} \sum_{j=0}^{n} \sqrt{7+\sqrt{13+4 \sqrt{3+2 \cos \alpha_{i}+2 \cos \beta_{j}+2 \cos \left(\alpha_{i}+\beta_{j}\right)}}} \\
& +\frac{\sqrt{3}+1}{6} \text {. }
\end{aligned}
$$

Consequently, one can easily arrive to the asymptotic value of incidence energy

$$
\begin{aligned}
& \lim _{m \rightarrow \infty} \lim _{n \rightarrow \infty} \frac{I E\left(J^{t}(m, n)\right)}{6(m+1)(n+1)} \\
& =\frac{1}{12} \int_{0}^{1} \int_{0}^{1} \sqrt{7-\sqrt{13-4 \sqrt{3+2 \cos \alpha_{i}+2 \cos \beta_{j}+2 \cos \left(\alpha_{i}+\beta_{j}\right)}}} d x d y \\
& +\frac{1}{12} \int_{0}^{1} \int_{0}^{1} \sqrt{7-\sqrt{13+4 \sqrt{3+2 \cos \alpha_{i}+2 \cos \beta_{j}+2 \cos \left(\alpha_{i}+\beta_{j}\right)}}} d x d y \\
& +\frac{1}{12} \int_{0}^{1} \int_{0}^{1} \sqrt{7+\sqrt{13-4 \sqrt{3+2 \cos \alpha_{i}+2 \cos \beta_{j}+2 \cos \left(\alpha_{i}+\beta_{j}\right)}}} d x d y \\
& +\frac{1}{12} \int_{0}^{1} \int_{0}^{1} \sqrt{7+\sqrt{13+4 \sqrt{3+2 \cos \alpha_{i}+2 \cos \beta_{j}+2 \cos \left(\alpha_{i}+\beta_{j}\right)}}} d x d y \\
& +\frac{\sqrt{3}+1}{6} \approx 1.3040 .
\end{aligned}
$$

The numerical integration value in last line is calculated with MATLAB software calculation.

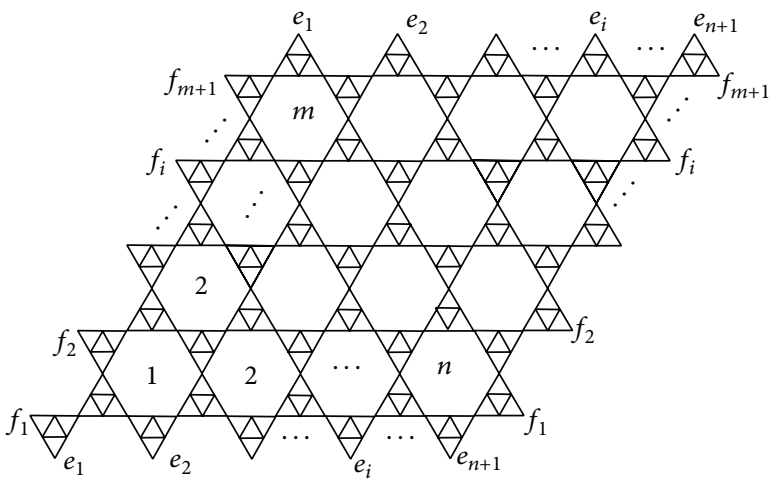

Figure 2: The $\operatorname{TKL}^{t}(m, n)$ lattice with toroidal boundary condition [5].

Hence $J^{t}(m, n)$ has the asymptotic incidence energy $\operatorname{IE}\left(J^{t}(m, n)\right) \approx 7.8240(m+1)(n+1)$.

2.2. The Triangular Kagomé Lattice. The triangular kagomé lattice [5] with toroidal boundary condition, denoted by $\operatorname{TKL}^{t}(m, n)$, is depicted in Figure 2 .

In order to obtain the $\operatorname{IE}(G)$ of toroidal boundary condition, we recall the spectrum and the Laplacian spectrum of $\mathrm{TKL}^{t}(m, n)$.

Theorem 4 (see [5]). The spectrum and the Laplacian spectrum of $\mathrm{TKL}^{t}(m, n)$ are

$$
\begin{aligned}
& \operatorname{Spec}_{A}\left(\operatorname{TKL}^{t}(m, n)\right) \\
& =\{\underbrace{-2,-2, \ldots,-2}_{3(m+1)(n+1)}, \underbrace{-1,-1, \ldots,-1}_{(m+1)(n+1)}, \underbrace{1,1, \ldots, 1}_{(m+1)(n+1)}\} \\
& \cup\left\{\frac{3 \pm \sqrt{13 \pm 4 \sqrt{3+2 \cos \alpha_{i}+2 \cos \beta_{j}+2 \cos \left(\alpha_{i}+\beta_{j}\right)}}}{2}\right\} \\
& \operatorname{Spec}_{L}\left(\operatorname{TKL}^{t}(m, n)\right) \\
& =\{\underbrace{6,6, \ldots, 6}_{3(m+1)(n+1)}, \underbrace{3,3, \ldots, 3}_{(m+1)(n+1)}, \underbrace{5,5, \ldots, 5}_{(m+1)(n+1)}\} \\
& \left.\cup \frac{5 \pm \sqrt{13 \pm 4 \sqrt{3+2 \cos \alpha_{i}+2 \cos \beta_{j}+2 \cos \left(\alpha_{i}+\beta_{j}\right)}}}{2}\right\}
\end{aligned}
$$

where $\alpha_{i}=2 \pi i /(m+1), \beta_{j}=2 \pi j /(n+1), i=0,1, \ldots, m$, $j=0,1, \ldots, n$.

Note that the triangular kagome lattice is the line graph of the 3.12.12 lattice and $\operatorname{TKL}^{t}(m, n)$ is a 4-regular graph with $9(m+1)(n+1)$ vertices. 
Consequently, we can easily get the signless Laplacian spectrum of $\operatorname{TKL}^{t}(m, n)$ :

$$
\begin{aligned}
& \operatorname{Spec}_{Q}\left(\operatorname{TKL}^{t}(m, n)\right) \\
& =\{\underbrace{2,2, \ldots, 2}_{3(m+1)(n+1)}, \underbrace{3,3, \ldots, 3}_{(m+1)(n+1)}, \underbrace{5,5, \ldots, 5}_{(m+1)(n+1)}\} \\
& \cup\left\{\frac{11 \pm \sqrt{13 \pm 4 \sqrt{3+2 \cos \alpha_{i}+2 \cos \beta_{j}+2 \cos \left(\alpha_{i}+\beta_{j}\right)}}}{2}\right\},
\end{aligned}
$$

where $\alpha_{i}=2 \pi i /(m+1), \beta_{j}=2 \pi j /(n+1), i=0,1, \ldots, m$, $j=0,1, \ldots, n$.
Theorem 5. Let $\alpha_{i}=2 \pi i /(m+1), \beta_{j}=2 \pi j /(n+1)$, $i=0,1, \ldots, m, j=0,1, \ldots, n$. Then the incidence energy of $\operatorname{TKL}^{t}(m, n)$ can be expressed as

$$
\operatorname{IE}\left(\operatorname{TKL}^{t}(m, n)\right)
$$

$=3 \sqrt{2}(m+1)(n+1)+(\sqrt{3}+\sqrt{5})(m+1)(n+1)$

$$
\begin{aligned}
& +\frac{1}{2} \sum_{i=0}^{m} \sum_{j=0}^{n} \sqrt{11-\sqrt{13-4 \sqrt{3+2 \cos \alpha_{i}+2 \cos \beta_{j}+2 \cos \left(\alpha_{i}+\beta_{j}\right)}}} \\
& +\frac{1}{2} \sum_{i=0}^{m} \sum_{j=0}^{n} \sqrt{11-\sqrt{13+4 \sqrt{3+2 \cos \alpha_{i}+2 \cos \beta_{j}+2 \cos \left(\alpha_{i}+\beta_{j}\right)}}} \\
& +\frac{1}{2} \sum_{i=0}^{m} \sum_{j=0}^{n} \sqrt{11+\sqrt{13-4 \sqrt{3+2 \cos \alpha_{i}+2 \cos \beta_{j}+2 \cos \left(\alpha_{i}+\beta_{j}\right)}}} \\
& +\frac{1}{2} \sum_{i=0}^{m} \sum_{j=0}^{n} \sqrt{11+\sqrt{13+4 \sqrt{3+2 \cos \alpha_{i}+2 \cos \beta_{j}+2 \cos \left(\alpha_{i}+\beta_{j}\right)}}} .
\end{aligned}
$$

Hence,

$$
\begin{aligned}
& \lim _{m \rightarrow \infty} \lim _{n \rightarrow \infty} \frac{\operatorname{IE}\left(\mathrm{TKL}^{t}(m, n)\right)}{9(m+1)(n+1)} \\
& =\lim _{m \rightarrow \infty} \lim _{n \rightarrow \infty} \frac{1}{18(m+1)(n+1)} \\
& \times \sum_{i=0}^{m} \sum_{j=0}^{n} \sqrt{11-\sqrt{13-4 \sqrt{3+2 \cos \alpha_{i}+2 \cos \beta_{j}+2 \cos \left(\alpha_{i}+\beta_{j}\right)}}} \\
& +\lim _{m \rightarrow \infty} \lim _{n \rightarrow \infty} \frac{1}{18(m+1)(n+1)} \\
& \times \sum_{i=0}^{m} \sum_{j=0}^{n} \sqrt{11-\sqrt{13+4 \sqrt{3+2 \cos \alpha_{i}+2 \cos \beta_{j}+2 \cos \left(\alpha_{i}+\beta_{j}\right)}}} \\
& +\lim _{m \rightarrow \infty} \lim _{n \rightarrow \infty} \frac{1}{18(m+1)(n+1)} \\
& \times \sum_{i=0}^{m} \sum_{j=0}^{n} \sqrt{11+\sqrt{13-4 \sqrt{3+2 \cos \alpha_{i}+2 \cos \beta_{j}+2 \cos \left(\alpha_{i}+\beta_{j}\right)}}} \\
& +\lim _{m \rightarrow \infty} \lim _{n \rightarrow \infty} \frac{1}{18(m+1)(n+1)} \\
& \times \sum_{i=0}^{m} \sum_{j=0}^{n} \sqrt{11+\sqrt{13+4 \sqrt{3+2 \cos \alpha_{i}+2 \cos \beta_{j}+2 \cos \left(\alpha_{i}+\beta_{j}\right)}}} \\
& +\frac{\sqrt{3}+\sqrt{5}}{9}+\frac{\sqrt{2}}{3} \\
& =\frac{1}{18} \int_{0}^{1} \int_{0}^{1} \sqrt{11-\sqrt{13-4 \sqrt{3+2 \cos \alpha_{i}+2 \cos \beta_{j}+2 \cos \left(\alpha_{i}+\beta_{j}\right)}}} d x d y
\end{aligned}
$$




$$
\begin{aligned}
& +\frac{1}{18} \int_{0}^{1} \int_{0}^{1} \sqrt{11-\sqrt{13+4 \sqrt{3+2 \cos \alpha_{i}+2 \cos \beta_{j}+2 \cos \left(\alpha_{i}+\beta_{j}\right)}}} d x d y \\
& +\frac{1}{18} \int_{0}^{1} \int_{0}^{1} \sqrt{11+\sqrt{13-4 \sqrt{3+2 \cos \alpha_{i}+2 \cos \beta_{j}+2 \cos \left(\alpha_{i}+\beta_{j}\right)}}} d x d y \\
& +\frac{1}{18} \int_{0}^{1} \int_{0}^{1} \sqrt{11+\sqrt{13+4 \sqrt{3+2 \cos \alpha_{i}+2 \cos \beta_{j}+2 \cos \left(\alpha_{i}+\beta_{j}\right)}}} d x d y \\
& +\frac{\sqrt{3}+\sqrt{5}}{9}+\frac{\sqrt{2}}{3} \approx 1.6390 .
\end{aligned}
$$

The above numerical integration value implies that $\operatorname{TKL}^{t}(m, n)$ has the asymptotic incidence energy $\operatorname{IE}\left(\operatorname{TKL}^{t}(m, n)\right) \approx 14.7510(m+1)(n+1)$.

Remark 6. In comparison to [5], the authors have derived the formulae of the number of spanning trees, the energy, and the Kirchhoff index of the triangular kagomé lattice with toroidal boundary condition in [5], while we have handled the $\operatorname{IE}(G)$ of the 3.12.12 lattice, triangular kagomé lattice, which enriches and extends the earlier results by Liu and Yan [5].

2.3. The $S^{t}(m, n)$ Lattice. The $S^{t}(m, n)$ lattice [20] with toroidal boundary condition, denoted by $S^{t}(m, n)$, can be constructed by starting with an $m \times n$ square lattice and adding two diagonal edges to each square, which are illustrated in Figure 3.

The eigenvalues of $A\left(S^{t}(m, n)\right)$ have been obtained in [20].

Lemma 7. The eigenvalues of $A\left(S^{t}(m, n)\right)$ are

$$
\begin{gathered}
2 \cos \frac{2 \pi i}{m}+2 \cos \frac{2 \pi j}{n}+4 \cos \frac{2 \pi i}{m} \cos \frac{2 \pi j}{n} \\
i=0,1, \ldots, m-1 ; \quad j=0,1, \ldots, n-1 .
\end{gathered}
$$

Notice that $S^{t}(m, n)$ is a 8-regular graph. Let $Q\left(S^{t}(m, n)\right)$ be the signless Laplacian matrix of $S^{t}(m, n)$, and then the signless Laplacian eigenvalues of $S^{t}(m, n)$ are

$$
\begin{array}{r}
8+2 \cos \frac{2 \pi i}{m}+2 \cos \frac{2 \pi j}{n}+4 \cos \frac{2 \pi i}{m} \cos \frac{2 \pi j}{n}, \\
i=0,1, \ldots, m-1 ; j=0,1, \ldots, n-1 .
\end{array}
$$

Based on Lemma 7 and the definition of the incidence energy, it is easy to deduce the following.

Theorem 8. Let $\alpha_{i}=2 \pi i / m, \beta_{j}=2 \pi j / n, i=0,1, \ldots, m-1$, $j=0,1, \ldots, n-1$, and then the incidence energy of $S^{t}(m, n)$ can be expressed as

$\operatorname{IE}\left(S^{t}(m, n)\right)$

$$
=\sum_{i=0}^{m-1} \sum_{j=0}^{n-1} \sqrt{8+2 \cos \frac{2 \pi i}{m}+2 \cos \frac{2 \pi j}{n}+4 \cos \frac{2 \pi i}{m} \cos \frac{2 \pi j}{n}} .
$$

Similarly, one can readily derive that

$$
\begin{aligned}
& \lim _{m \rightarrow \infty} \lim _{n \rightarrow \infty} \frac{\operatorname{IE}\left(S^{t}(m, n)\right)}{m n} \\
& =\lim _{m \rightarrow \infty} \lim _{n \rightarrow \infty} \frac{1}{m n} \sum_{i=0}^{m-1} \sum_{j=0}^{n-1} \sqrt{8+2 \cos \frac{2 \pi i}{m}+2 \cos \frac{2 \pi j}{n}+4 \cos \frac{2 \pi i}{m} \cos \frac{2 \pi j}{n}} \\
& =\int_{0}^{1} \int_{0}^{1} \sqrt{8+2 \cos 2 \pi x+2 \cos 2 \pi y+4 \cos 2 \pi x \cos 2 \pi y} d x d y \\
& =\frac{1}{4 \pi^{2}} \int_{0}^{2 \pi} \int_{0}^{2 \pi} \sqrt{8+2 \cos x+2 \cos y+4 \cos x \cos y} d x d y \\
& \approx 2.7883 .
\end{aligned}
$$




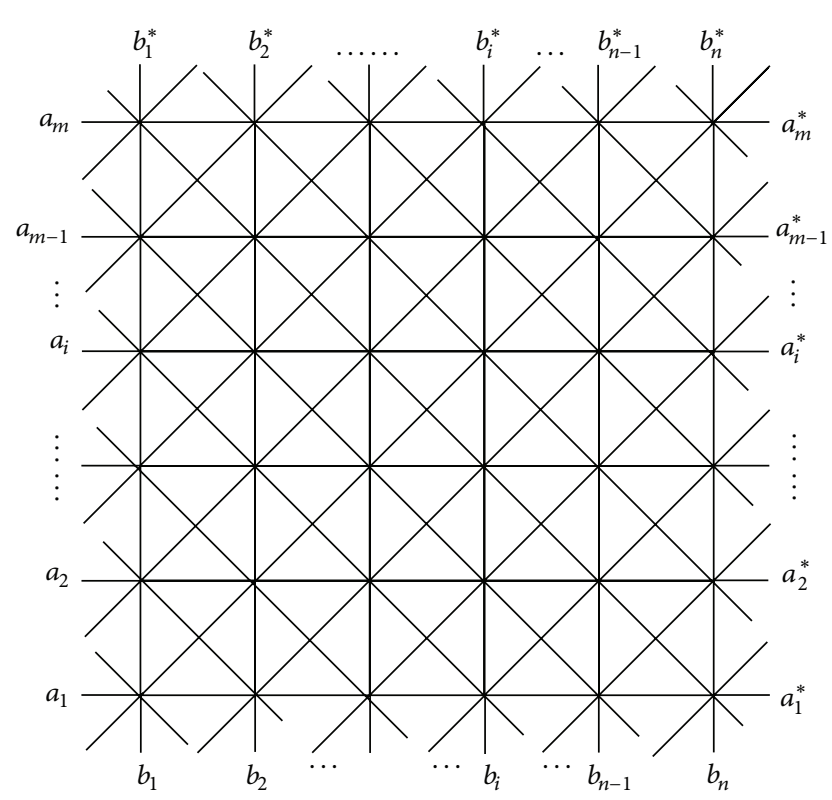

FIGURE 3: The $S^{t}(m, n)$ lattice with toroidal boundary condition [20].

The above numerical integration value implies that $S^{t}(m, n)$ has the asymptotic incidence energy $\operatorname{IE}\left(S^{t}(m, n)\right) \approx$ $2.7883 \mathrm{mn}$. Summing up, we complete the proof.

\section{Remarking Conclusions}

In this paper, we deduced the formulae and asymptotic formulae expressing the incidence energy of the 3.12.12 lattice, triangular kagomé lattice, and $S(m, n)$ lattice with toroidal boundary condition, respectively.

It is well known that dealing with the problem of the asymptotic incidence energy of various lattices with the free boundary is not an easy task; however, we can convert the more difficult problems to relatively simple ones via the applications of analysis approach with the help of calculational software. In fact, our approach can be used widely to handle the asymptotic behavior of other lattices and can obtain some useful results simultaneously.

\section{Conflict of Interests}

The authors declare that there is no conflict of interests regarding the publication of this paper.

\section{Acknowledgments}

The work of J. B. Liu is partly supported by the Natural Science Foundation of Anhui Province of China under Grant no. KJ2013B105, and the National Science Foundation of China under Grant nos. 11471016, and 11401004. The work of J. Xie was funded by the Natural Science Foundation of Anhui Province of China under Grant no. 1208085MA15 and the Key Project Foundation of Scientific Research, Education Department of Anhui Province, under Grant no. KJ2014ZD30 and the Key Construction Disciplines Foundation of Hefei University under Grant no. 2014XK08.

\section{References}

[1] I. Gutman, "The energy of a graph: old and new results," in Alg ebraic Combinatorics and Appli cations, pp. 196-211, Springer, Berlin, Germany, 2001.

[2] V. Nikiforov, "The energy of graphs and matrices," Journal of Mathematical Analysis and Applications, vol. 326, no. 2, pp. 1472-1475, 2007.

[3] W. Yan and L. Ye, "On the minimal energy of trees with a given diameter," Applied Mathematics Letters, vol. 18, no. 9, pp. 10461052, 2005.

[4] W. G. Yan and Z. H. Zhang, "Asymptotic energy of lattices," Physica A, vol. 388, no. 8, pp. 1463-1471, 2009.

[5] X. Y. Liu and W. G. Yan, "The triangular kagomé lattices revisited," Physica A: Statistical Mechanics and its Applications, vol. 392, no. 22, pp. 5615-5621, 2013.

[6] F. Y. Wu, "Dimers on two-dimensional lattices," International Journal of Modern Physics, vol. 20, no. 32, pp. 5357-5371, 2006.

[7] R. Shrock and F. Y. Wu, "Spanning trees on graphs and lattices in $d$ dimensions," Journal of Physics A: Mathematical and General, vol. 33, no. 21, pp. 3881-3902, 2000.

[8] Z. Zhang, "Some physical and chemical indices of cliqueinserted lattices," Journal of Statistical Mechanics: Theory and Experiment, vol. 10, Article ID P10004, 2013.

[9] J. B. Liu, X. F. Pan, J. Cao, and F. F. Hu, "A note on some physical and chemical indices of clique-inserted lattices," Journal of Statistical Mechanics: Theory and Experiment, Article ID P06006, 2014.

[10] D. Yao, Y. L. Loh, E. W. Carlson, and M. Ma, "XXZ and Ising spins on the triangular kagome lattice," Physical Review B, vol. 78, no. 2, Article ID 024428, 2008.

[11] J. Strečka, L. Čanová, M. Jaščur, and M. Hagiwara, "Exact solution of the geometrically frustrated spin-1/2 Ising-Heisenberg model on the triangulated kagome (trianglesin- triangles) lattice," Physical Review B, vol. 78, no. 2, Article ID 024427, 2008.

[12] B. Mohar and Y. Alavi, "The Laplacian spectrum of graphs," in Graph Theory, Combinatorics, and Applications, vol. 2, pp. 871898, 1991.

[13] D. Cvetković and P. Rowlinson, "Signless Laplacians of finite graphs," Linear Algebra and Its Applications, vol. 423, no. 1, pp. 155-171, 2007.

[14] F. Zhang, Y. Chen, and Z. Chen, "Clique-inserted-graphs and spectral dynamics of clique-inserting," Journal of Mathematical Analysis and Applications, vol. 349, no. 1, pp. 211-225, 2009.

[15] M. R. Jooyandeh, D. Kiani, and M. Mirzakhah, "Incidence energy of a graph," MATCH Communications in Mathematical and in Computer Chemistry, vol. 62, no. 3, pp. 561-572, 2009.

[16] I. Gutman, D. Kiani, and M. Mirzakhah, "On incidence energy of graphs," MATCH: Communications in Mathematical and in Computer Chemistry, vol. 62, no. 3, pp. 573-580, 2009.

[17] I. Gutman, D. Kiani, M. Mirzakhah, and B. Zhou, "On incidence energy of a graph," Linear Algebra and its Applications, vol. 431, no. 8, pp. 1223-1233, 2009.

[18] K. C. Das and I. Gutman, "On incidence energy of graphs," Linear Algebra and Its Applications, vol. 446, no. 1, pp. 329-344, 2014.

[19] L. Ye, "On the Kirchhoff index of some toroidal lattices," Linear and Multilinear Algebra, vol. 59, no. 6, pp. 645-650, 2011.

[20] L. Ye, "The energy of a type of lattice," Applied Mathematics Letters, vol. 24, no. 2, pp. 145-148, 2011. 


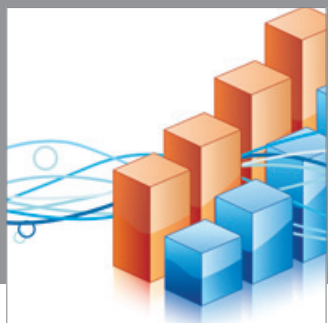

Advances in

Operations Research

mansans

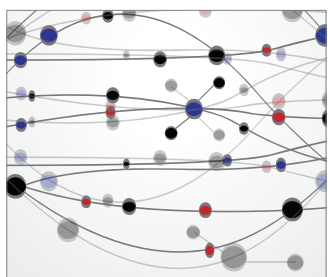

The Scientific World Journal
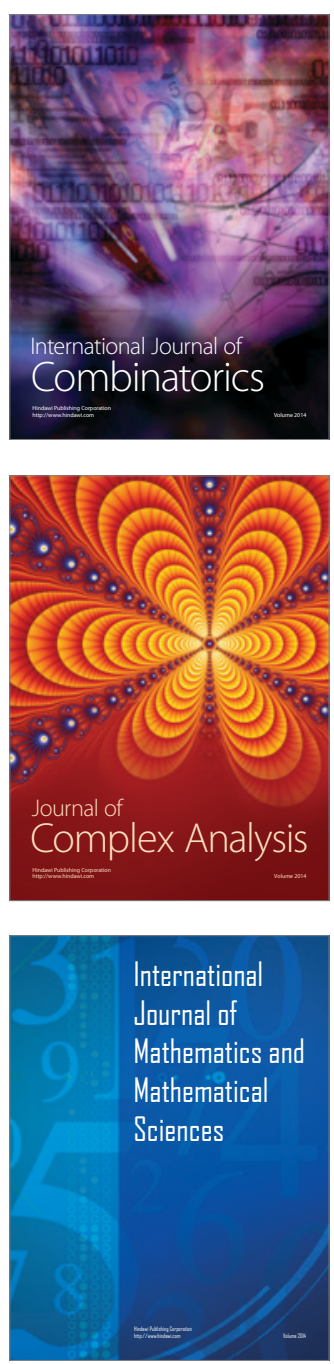
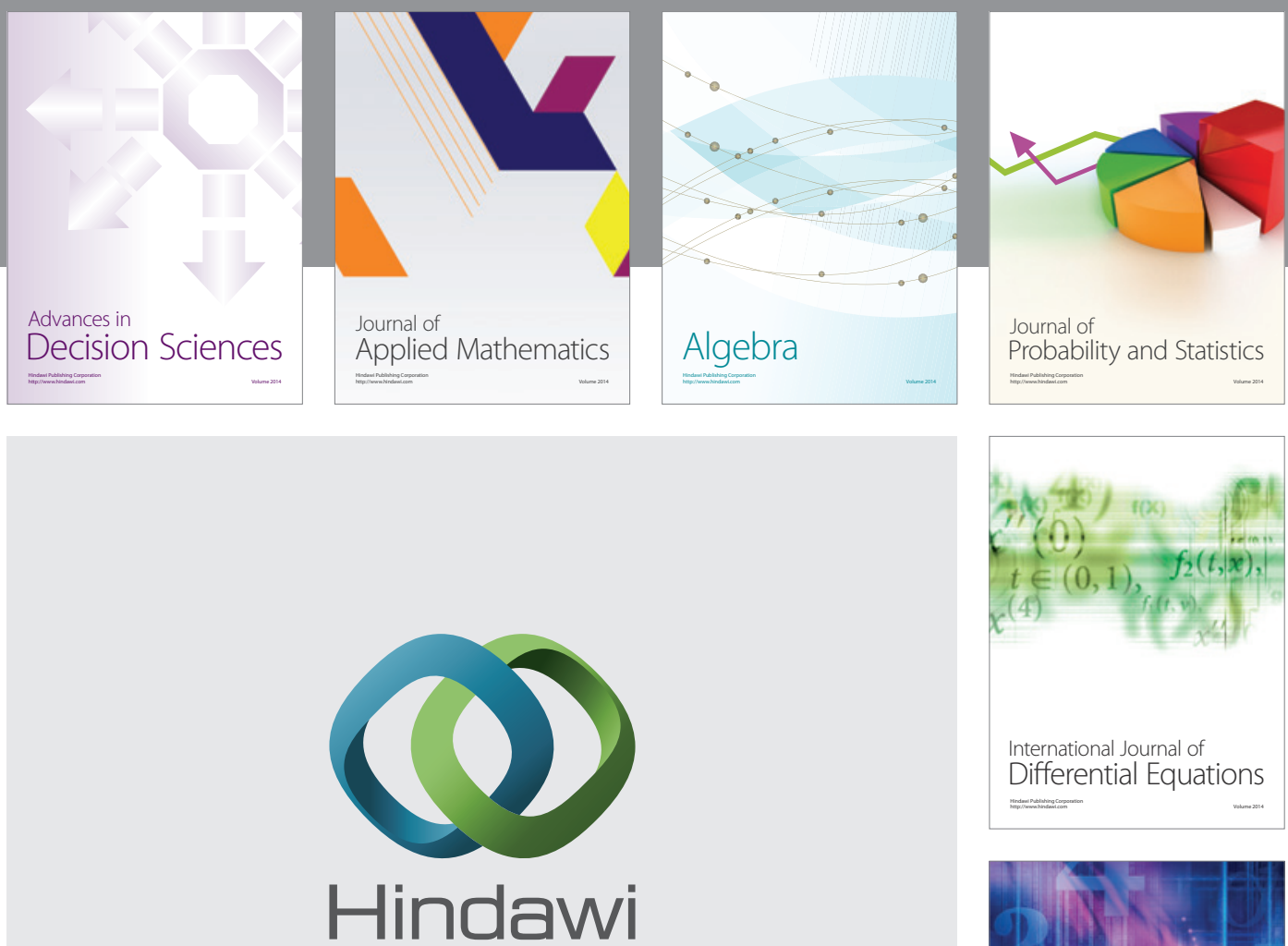

Submit your manuscripts at http://www.hindawi.com
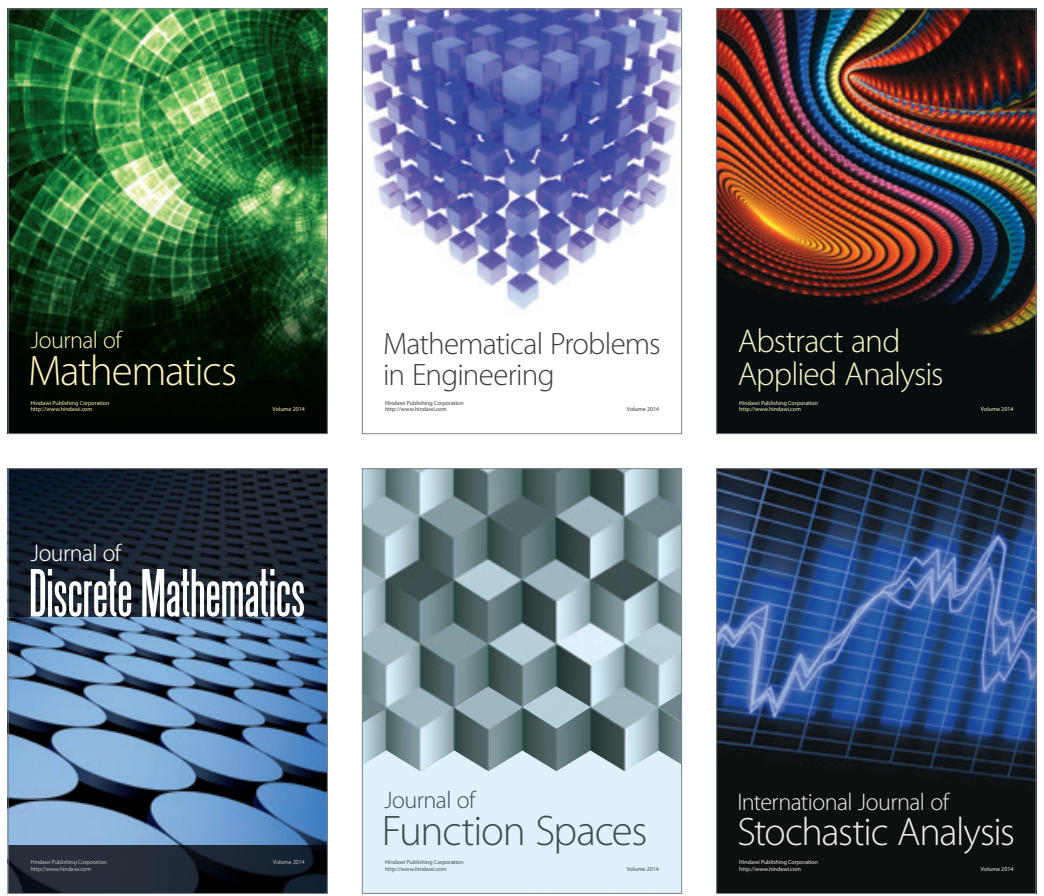

Journal of

Function Spaces

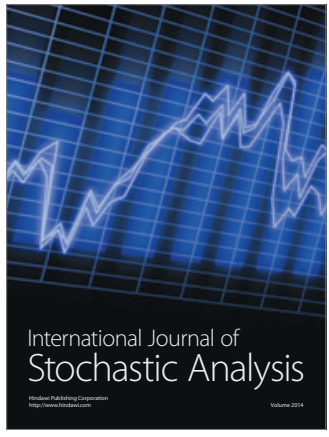

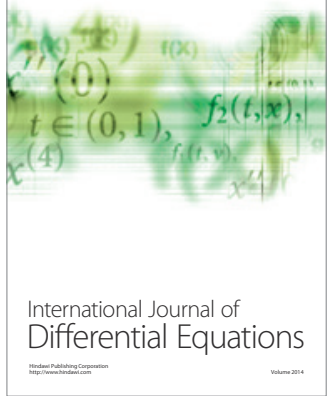
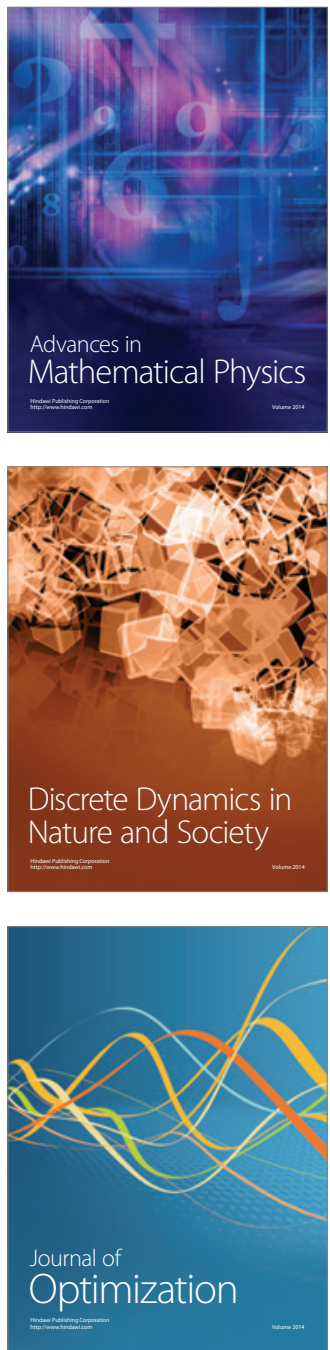